\title{
RF FOCUSSING - AN INSTRUMENT FOR BEAM QUALITY IMPROVEMENT IN SUPERCONDUCTING RF GUNS
}

\author{
V. Volkov, Budker INP, 630090, Lavrentev Ave., 11, Novosibirsk, Russia, VNVolkov@ inp.nsk.su, \\ D. Janssen, FZ Rossendorf, Postfach 5101 19, D-01314 Dresden, Germany
}

\begin{abstract}
$\mathrm{Rf}$ electron guns require an external magnetic field focussing to produce a low emittance electron beam. A dc magnetic field focuses electrons in the beam already inside the cavity as they leave the photocathode. In superconducting rf guns (srf) the possibility of external magnetic focussing is quite limited. In this paper we investigate an effect of rf focussing in the first half cell of a $3+1 / 2$ cell srf gun cavity without a dc magnetic focussing. The shape of the first half cell is optimized. The calculations were done to fulfil the beam requirements of the injector for the ELBE project [1]. Besides normalized transversal emittances of the order of $1 \pi \mathrm{mm} \cdot \mathrm{mrad}$ for bunch charges up to $1 \mathrm{nC}$ were obtained. In the paper a graphic explanation of the rf focusing effect is represented.
\end{abstract}

\section{INTRODUCTION}

Photocathode rf guns are today the most advanced type of electron injectors for high brightness accelerators [3]. Would be better to combine the high brightness of rf guns with low rf losses of superconducting cavities. A disadvantage of superconducting technology is its sensitivity to external magnetic fields. So it becomes impossible to focus the electron beam by an external magnetic field inside a superconducting cavity. In this paper we calculate the beam parameters of superconducting $\mathrm{rf}$ guns with rf focussing only, while no external magnetic field applied. RF focussing is achieved by pulling the cathode out of the cavity behind the back wall plane. Extensive beam dynamics simulations for different bunch charges were made. Explanation of $\mathrm{rf}$ focussing effect were made too.

\section{GEOMETRY OF RF GUN CAVITY}

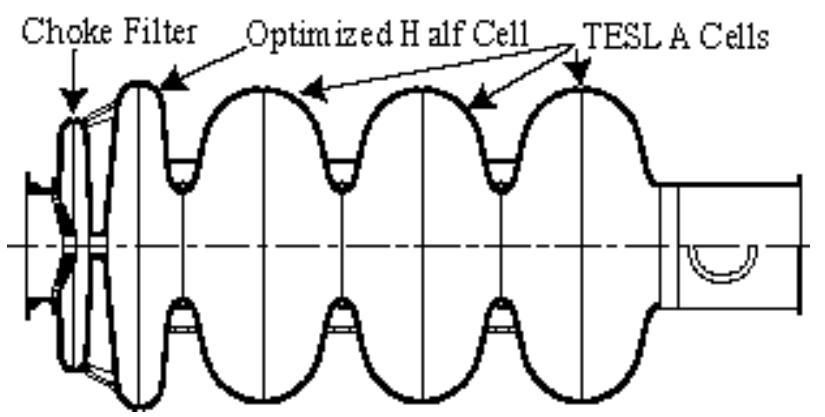

Figure 1: Superconducting rf gun cavity layout.
The rf gun cavity for the proposed superconducting injector is shown in fig.1. It consists of three TESLA cells and a half cell. A $\varnothing 10 \mathrm{~mm}$ copper cathode with a photolayer on its tip is located somewhat at the half cell back wall plane, inside a $\emptyset 12 \mathrm{~mm} \mathrm{Nb}$ tube which connects the cavity to the choke flange filter. The rf choke filter prevents rf power leakage from the cavity to the cathode holder.

\section{RF FOCUSING FIELD}

We need an electric rf field with sufficiently large radial component near the cathode in order to focus the electron beam. Such field pattern can be obtained by pulling the cathode stem out of the cavity toward the choke filter by the distance $\Delta \mathrm{z}$ behind the half cell back wall plane. The resulting field distribution is given in fig.2.

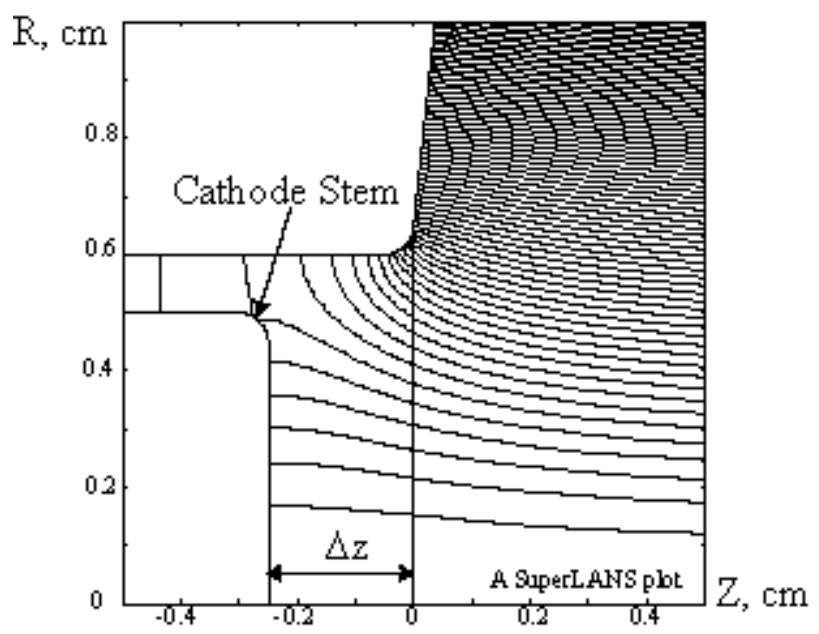

Figure 2: Close view of the field pattern near the cathode.

The accelerating component $E_{z}$ starts with the value $E_{c}$ at the cathode and reaches its maximum $\mathrm{E}_{\mathrm{z} \max }$ Using the Maxwell equation, one obtains ( $\mathrm{R}$ is the tube radius)

$$
\left|\mathrm{E}_{\mathrm{r} \max }\right| / \mathrm{r} \leq 2 \cdot\left(\mathrm{E}_{\mathrm{Zmax}}-\mathrm{E}_{\mathrm{c}}\right) / \pi \cdot \mathrm{R}
$$

Increasing $\Delta \mathrm{z}$ we would further increase $\mathrm{E}_{\mathrm{r}}$ but, at the same time, decrease $\mathrm{E}_{\mathrm{C}}$. In principle at the cathode we need large accelerating component $\mathrm{E}_{\mathrm{Z}}$ and a certain radial focussing field $\mathrm{E}_{\mathrm{r}}$. The optimal value of $\Delta \mathrm{z}$ is $\Delta \mathbf{z}=\mathbf{2 . 5} \mathbf{~ m m}$, nearly independent from the bunch charge. 


\section{CALCULATION OF THE BEAM PROPERTIES}

The beam parameters were calculated using the particle tracking code PARMELA [5]. In the simulations a bunch of 10,000 charged particles was used. RF fields distribution was calculated with SuperLANS code [4]. Further decrease of transversal emittances has been obtained, assuming a superposition of three Gaussian functions for the laser pulse. The distance between the centers of each two adjacent Gaussian pulses is $2 \sigma$, where $\sigma$ is the longitudinal Gaussian width. In many runs of the program we varied the following parameters in order to find the optimal result:

- the injection phase of the laser pulse

- the displacement $\Delta \mathrm{z}$ of the cathode behind the half cell back wall plane

- the longitudinal and transversal shape parameter of the laser pulse, which determines the bunch shape at the cathode.

The results of the calculations along with the optimal parameter set are presented in table 1

\section{DISCUSSION OF THE RESULTS}

For the charges of $85 \mathrm{pC}$ and $200 \mathrm{pC}$ it is quite easy to obtain normalized transversal emittances lower than $1 \pi \mathrm{mm} \cdot \mathrm{mrad}$, which is quite sufficient for infrared FEL applications. Thus the superconducting rf gun is also an ideal injector for the ELBE project [1] and for high power FELs, which are under development in CEBAF [6].

For the charge of $1 \mathrm{nC}$ we obtain a transversal emittance of $0.98 \pi \mathrm{mm} \cdot \mathrm{mrad}$ and a longitudinal emittance of $22.3 \mathrm{~mm} \cdot \mathrm{keV}$ for the bunch, head and tail truncated by $10 \%$ of particles - here there are low density slices of bunch which strongly deteriorate it's quality (a transversal emittance of $2.28 \pi \mathrm{mm} \cdot \mathrm{mrad}$ and a longitudinal emittance of $35.8 \mathrm{~mm} \cdot \mathrm{keV}$ for the full bunch). The analysis of structure of particles in the full bunch has shown, that $96 \%$ of particles form a transversal emittance by $1 \pi \mathrm{mm} \cdot \mathrm{mrad}$. These values are close to those, needed for the TESLA Test Facility (TTF)[2].

In these calculations the influence of the rf focussing forces can be clearly demonstrated. Without an $\mathrm{rf}$ focussing $(\Delta z=0)$ the minimal transversal emittance increases by a factor larger than three.

The analysis of the PARMELA calculations using a quasilaminar approach [9] have shown, that there are two main causes of improving of bunch quality when we use an rf focusing in the first half cell, as follows:

- decreasing of average bunch radius in rf gun (see fig.3);

- leveling of a charge density on bunch length (fig.5).

\subsection{Decreasing of average bunch radius}

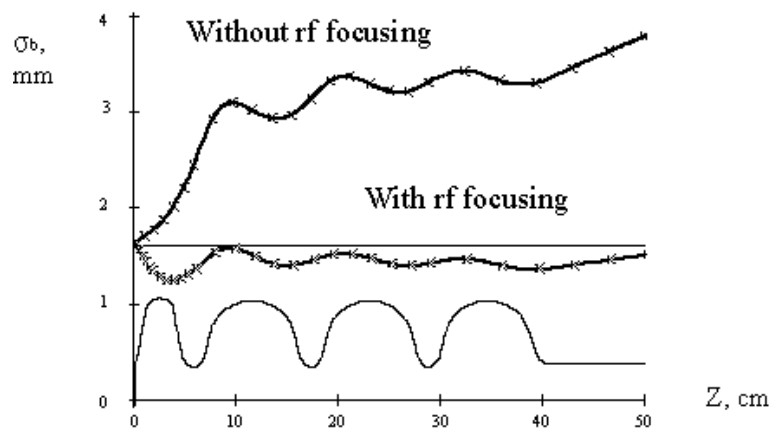

Fig.3: Rms bunch radius $\sigma_{\mathrm{b}}$ vs distance from cathode

Table 1: Results of PARMELA calculations for the $3+1 / 2$ cell $1.3 \mathrm{GHz}$ superconducting rf gun

\begin{tabular}{|l|l|l|l|l|l|l|l|}
\hline \multicolumn{1}{|c|}{ Parameter } & \multicolumn{3}{c|}{ Units } & \multicolumn{3}{c|}{ Full bunch / 10\% head\&tail truncated } & $\Delta \mathrm{z}=0$ \\
\hline Bunch charge & $\mathrm{pC}$ & 85 & 200 & 1000 & 200 & 1000 & 1000 \\
\hline Laser pulse shape & Gaussians number $\times \sigma(\mathrm{ps})$ & $1 \times 2.3$ & $1 \times 3.0$ & $1 \times 3.0$ & $3 \times 3.0$ & $3 \times 3.0$ & $3 \times 3.0$ \\
\hline Laser pulse length $(\mathrm{FWHM})$ & $\mathrm{ps}$ & 5.3 & 7 & 7 & 18 & 18 & 18 \\
\hline Laser spot diameter & $\mathrm{mm}$ & 3 & 3 & 4 & 3 & 4.6 & 4.6 \\
\hline Accelerating gradient & $\mathrm{MV} / \mathrm{m}$ & 20 & 25 & 25 & 25 & 25 & 25 \\
\hline Injection phase & $\mathrm{deg}$ & 12.5 & 33 & 30 & 33 & 30 & 51.5 \\
\hline Distance from the cathode & $\mathrm{cm}$ & 150 & 230 & 190 & 110 & 150 & 50 \\
\hline Energy & $\mathrm{MeV}$ & 7.912 & 10.00 & 9.986 & 10.01 & 10.00 & 10.01 \\
\hline Energy spread (rms) & $\mathrm{keV}$ & 3.786 & 5.78 & 9.82 & 9.85 & 17.07 & 12.9 \\
& & 2.964 & 4.31 & 8.43 & 7.49 & 12.53 & 9.8 \\
\hline Transversal normalized & $\pi \mathrm{mm} \cdot \mathrm{mrad}$ & 0.86 & $\mathbf{1 . 0 7}$ & 2.2 & $\mathbf{1 . 0 6 5}$ & 2.28 & 5.85 \\
emittance (rms) & & 0.427 & 0.464 & 2.07 & 0.42 & $\mathbf{0 . 9 7 6}$ & 4.18 \\
\hline Longitudinal emittance $(\mathrm{rms})$ & $\mathrm{mm} \cdot \mathrm{keV}$ & 2.046 & 3.598 & 15.96 & 15.5 & 35.8 & 23.36 \\
& & 0.719 & 2.12 & 9.84 & 9.6 & 22.32 & 15.45 \\
\hline Bunch radius $(\mathrm{rms})$ & $\mathrm{mm}$ & 1.76 & 2.65 & 6.93 & 0.63 & 2.9 & 3.71 \\
& & 1.845 & 2.786 & 7.22 & 0.61 & 2.9 & 3.7 \\
\hline Bunch length $(\mathrm{rms})$ & $\mathrm{mm}$ & 0.576 & 0.952 & 1.7 & 1.61 & 2.1 & 1.83 \\
& & 0.486 & .821 & 1.48 & 1.4 & 1.84 & 1.58 \\
\hline
\end{tabular}


As for each slice of a beam, there is a shift of rf field phase, the action of field on them is be unequal therefore their phase space angles are differ from each other. But this differences (bunch emittance identical) vanish when the radius is smaller. Because at smaller radius the focusing and defocusing effect of radial components of an accelerating field in each cell becomes smaller, as the fig.4 indicates. Such conclusion was confirmed by our first calculations [7] where a bunch charge is neglected.

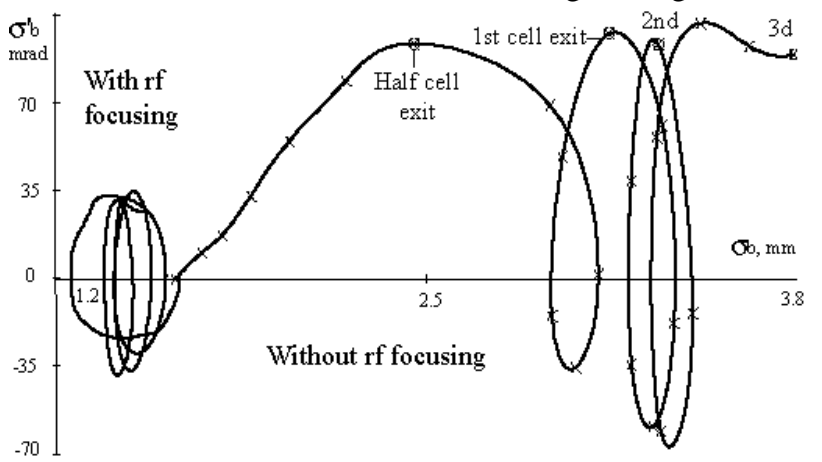

Fig.4: Bunch divergence $\sigma_{b}{ }_{b}=\left(d \sigma_{b} / d z\right) \cdot \beta \cdot \gamma$ vs $\sigma_{b}(r m s)$

\subsection{Leveling of a charge density}

A less dense slices of a bunch (head and tail as a rule) are compressed by rf focusing stronger. As a result the density of these low density slices is risen. Through such levelling of a charge density, the bunch emittance is decreased but under the influence of one's own momentum has a minimum far after the gun exit. In the following we will confirm it by solving the model of Ref. $[8,9]$. In this model the envelope equation for an axial slice of the bunch is given by

$$
\mathrm{d}^{2} \sigma / \mathrm{dt}^{2}+\mathrm{K} \sigma-\mathrm{K}_{\mathrm{S}} / \sigma=0
$$

where we have adopted the notation of $[8,9] . \sigma$ is the r.m.s. slice radius, $K$ represents the effect of external focussing and $\mathrm{K}_{\mathrm{s}}$ characterize the space charge force. In eq.(2) we approximate the radial focussing rf field by a constant positive $\mathrm{K}$ value in a small region after the cathode and by $\mathrm{K}=0$ outside of this region. With this approximation the solution of eq.(2) for two different charge densities of the slices $\left(\mathrm{K}_{\mathrm{s}, 1}=0.5^{*} \mathrm{~K}_{\mathrm{s}, 2}\right)$ in the transversal phase space $\left(\sigma, \sigma^{\prime}\right)$ is represented in fig. 5 .

The slices starts with the same radius $\sigma_{o}$ and zero divergence. Due to the space charge forces and under the influence of one's own momentum at the point 3-3' the total emittance of the bunch (for the made assumptions) goes to zero.

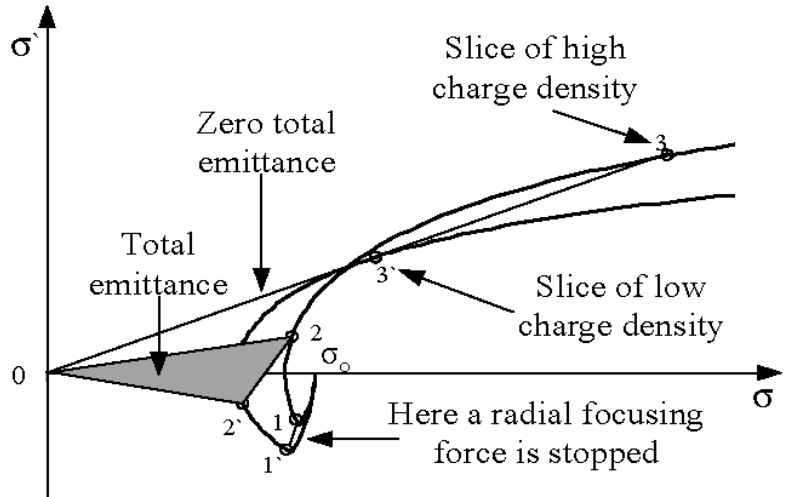

Fig.5: Solution of equation (1) in transversal phase space $\left(\sigma^{`}=\mathrm{d} \sigma / \mathrm{dz}\right) . \sigma$ of less dense slice is compressed stronger.

\section{CONCLUSION}

Using the PARMELA code we optimized the beam parameters at the output of a superconducting $\mathrm{rf}$ gun for bunch charges of $85 \mathrm{pC}, 200 \mathrm{pC}$ and $1 \mathrm{nC}$. A realistic geometry of the first half cell of the gun cavity has been used. A specially designed field profile near the cathode is used. The focussing is ensured by radial components of electric field. The simple explanation of the rf focusing effect in the first half cell have been done. The results makes us optimistic about the application of superconducting rf gun as an injector of high quality beam in compact superconducting linacs for FEL's.

\section{REFERENCES}

[1] F.Gabriel ed.., Internal design report, Forschungszentrum Rossendorf, Dresden 1995

[2] D.A.Edwards, TESLA Test Facility - Design report, Technical report, DESY, Hamburg 1995

[3] Chr. Travier, NIM A304 (1991) 285

[4] D.G.Myakishev, V.P.Yakovlev, in Proc. PAC 1991 Vol.5, p.3002

[5] J.Billen, L. Young, in Proc. PAC 1993 Vol.2, p.790

[6] H.F.Dylla, in Proc. PAC 1995, Dallas 1995

[7] V.Volkov et al., in Proc. PAC 1997, vol. 2, p.2796

[8] B.E.Carlsten, D.T.Palmer, NIM A425 (1999) 37

[9] L.Serafini, J.B.Rosenzweig, Phys.Rev. E55(1997)7565 